\title{
Prevalence and Correlates of Gastroesophageal Reflux Disease in Southern Iran: Pars Cohort Study
}

\author{
Zohre Khodamoradi ${ }^{1}$, Abdullah Gandomkar ${ }^{2}$, Hossein Poustchi ${ }^{3}$, Alireza Salehi ${ }^{4}$, \\ Mohammad Hadi Imanieh ${ }^{5,}$, Arash Etemadi ${ }^{3,6}$, Reza Malekzadeh ${ }^{2,3}$
}

1. MPH Department, Student Research Committee, Shiraz Medical School, Shiraz University of Medical Sciences, Shiraz, Iran.

2. Non-Communicable Disease Research Center, Shiraz University of Medical Sciences, Shiraz, Iran.

3. Digestive Disease Research Center, Digestive Disease Research Institute, Tehran University of Medical Sciences, Tehran, Iran.

4. MPH Department, Shiraz University of Medical Sciences, Shiraz, Iran.

5. Section of Gastroenterology, Department of Pediatrics, Nemazee Hospital, Shiraz University of Medical Sciences, Shiraz, Iran.

6. Division of Cancer Epidemiology and Genetics, National Center Institute, NIH, Bethesda, Maryland, USA.

\section{* Corresponding Author:}

Mohammad Hadi Imanieh, MD Department of Pediatrics, Nemazee Hospital, Shiraz University of Medical Sciences, Shiraz, Iran.

Tel: + 987132332366

Fax: +987132359317

Email: imaniehm@sums.ac.ir

Received: 07 Mar. 2017

Accepted: 26 May 2017

\section{ABSTRACT}

\section{BACKGROUND}

Prevalence of gastroesophageal reflux disease (GERD) is increasing worldwide. We aimed to estimate the prevalence of GERD in Pars Cohort Study (PCS) and to find its correlates.

\section{METHODS}

We used the baseline data from PCS. PCS was conducted in the district of Valashahr in Fars province in southern Iran from 2012 to 2014. 9264 inhabitants who were 40-75 years old, and agreed to participate were enrolled. Data were collected by a structured questionnaire and simple physical examination of all participants.

\section{RESULTS}

Generally, 58.50\% (95\% CI 57.49 - 59.51) of the participants had GERD and $25.10 \%$ (95\% CI 24.22 - 25.99) experienced it at least weekly. Approximately, $32.0 \%, 52.0 \%$, and $24.4 \%$ of the participants reported heart burn sensation, regurgitation, and both symptoms, respectively. Being female (OR: 1.45, 95\% CI 1.27 - 1.65), being older (OR: 1.20, 95\% CI $1.06-1.36$ ), being divorced/ widowed/separated (OR: 1.38, 95\% CI 1.01 - 1.91), and lower education (OR: 1.43, 95\% CI 1.02 2.03) were associated with frequent GERD.

\section{CONCLUSION}

GERD is common in PCS and its prevalence is close to that in western countries. Being female, higher age, being divorced/widowed/separated, lower education, history of hypertension, anxiety, insomnia, and non-cigarette tobacco smoking were associated with frequent GERD. We are going to investigate the causal relationship between these risk factors and GERD in the next stages of PCS.

\section{KEYWORDS:}

Epidemiology; Gastroesophageal reflux; Heartburn; Regurgitation; Pars cohort study; Iran.

Please cite this paper as:

Khodamoradi Z, Gandomkar A, Poustchi H, Salehi AR, Imanieh MH, Etemadi A, Malekzadeh R. Prevalence and Correlates of Gastroesophageal Reflux Disease in Southern Iran: Pars Cohort Study. Middle East J Dig Dis 2017;9:129-138. DOI: 10.15171/mejdd.2017.63.

\section{INTRODUCTION}

Gastroesophageal reflux disease (GERD) has been increasing across the world in the past decades. ${ }^{1-3}$ It is especially common in western countries, ${ }^{1,4,5}$ and is the most frequent diagnosis in patients with gastrointestinal problems. Age, sex, life style, medication, pregnancy, and geographic variation have been shown to be associated with GERD. ${ }^{6-9}$

The prevalence of GERD is low in East Asia, but it is more prevalent in the Middle East, where the prevalence is similar to many western countries. About 18.1 -27.8\% in North America, 23.0\% in South America, $8.8-25.9 \%$ in Europe, $11.6 \%$ in Australia, $2.5-7.8 \%$ in East Asia, and 8.7-33.1\% in the Mid- 
dle East experience GERD symptoms. , $^{2,8,10-14}$ Delavari and colleagues found that the prevalence of GERD in Iran is $21.2 \% .{ }^{11}$ A study on the Qashqai migrating nomads of Fars province revealed that $33.1 \%$ of them had at least weekly GERD symptoms. ${ }^{15}$ This showed a higher prevalence of GERD compared with other studies in Iran, which may not be the representative of the whole country. ${ }^{12,13,15}$ These differences may be due to different definitions of GERD and lack of a gold standard test for diagnosing it. Most of previous studies have considered heartburn and/ or regurgitation in participants as GERD. ${ }^{12,14,16-23}$

Burning sensation (heartburn) and regurgitation of gastro-duodenal contents into the esophagus are the

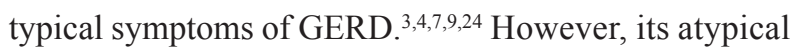
symptoms are cough, asthma, chest pain, and hoarseness. ${ }^{3,9,25-27}$ GERD has also several serious complications such as esophageal ulcer, stricture, Barrett's esophagus, and esophageal adenocarcinoma. ${ }^{2,6,10}$ Both of its symptoms and complications affect the patients' quality of life., ${ }^{428}$

Due to different reports of epidemiological studies, increase in the trend of GERD, and lack of a large study in the south of Iran, we aimed to determine the prevalence and associated risk factors of this disease, using a cross-sectional analysis of the data obtained during Pars Cohort Study (PCS).

\section{MATERIALS AND METHODS \\ Subjects}

PCS has been designed as a prospective study of the burden and risk factors for non-communicable diseases in Fars province in southern Iran. The site of this study is Valashahr District with 40000 residents. The inhabitants of the region consist of a variety of ethnicities including Fars, Turk, and others. All 9721 inhabitants aged between 40 - 75 years were invited, but those who were unwilling to participate or those who were a temporary resident were excluded. Finally, 9264 participants were enrolled in this study from 2012 to 2014. Details of the study design have been published earlier. ${ }^{29}$

\section{Ethics Statement}

The study protocol was approved by the Ethics Committees of Tehran University of Medical Sciences and Shiraz University of Medical Sciences. The purpose and the method of the study were explained to the participants if they were illiterate. The participants were invited to visit the center and participate in the study if they agreed. Informed consents were signed by the participants.

\section{Measurement of variables and definitions}

We used baseline PCS data in our cross-sectional study. Data were collected through a structured questionnaire and simple physical examination. The data on demographic characteristics, lifestyle factors, disease history, family history, medication history, smoking, and the abuse of opium and alcohol were collected. The participants were asked about GERD symptoms including heartburn (a burning sensation behind the sternum) and regurgitation (regurgitation of gastroduodenal contents into the esophagus) over the past year and severity and frequency of these symptoms. The frequency of the symptoms was categorized into: one to 10 times per year, once a month, two or three times a month, weekly, and daily. GERD was defined as at least one participant's recall of heartburn and/or regurgitation during the past 12 months and frequent GERD was having at least weekly symptoms. The severity of the symptoms was recorded as mild, moderate, severe, and very severe. Mild means that participants do not feel GERD symptoms if they do not think about it. Moderate means symptoms not interfering with daily work. Severe symptom means that it interferes with daily work, and very severe symptom means it affects the life and bothers the participants.

Height and weight were measured by a trained health worker according to a standard protocol. Body mass index (BMI) was calculated as weight in kilograms divided by squared height in meters. BMI was classified using the WHO classification as underweight (lower than 18.5), normal (18.5 - 24.9), overweight (25 - 29.9), and obese (30 or more). The waist circumference more than 102 centimeters for men and 88 centimeters for (non-pregnant) women and also waist to hip ratio above 0.90 for men and 0.85 for women were considered as central adiposity. ${ }^{30,31}$

Positive opium abuse (teriak, heroin, sukhteh, and shireh), non-cigarette tobacco (hookah, nass, and pipe), and cigarette were defined as at least once per week for the past six months, and positive alcohol use was defined as drinking once per month for the past six months. 
Table 1: Frequency and severity of heart burn sensation and regurgitation in participants of Pars Cohort Study in the last 12 months (n=9264)

\begin{tabular}{|c|c|c|c|c|c|c|c|c|c|c|}
\hline \multirow{3}{*}{ Symptom } & \multicolumn{5}{|c|}{ Frequency } & \multicolumn{5}{|c|}{ Severity } \\
\hline & $1-10 /$ year & $1 /$ month & 2-3/month & Weekly & Daily & Mild & Moderate & Severe & Very severe & Total \\
\hline & N (\%) & N (\%) & N (\%) & N (\%) & N (\%) & $\mathrm{N}(\%)$ & $\mathrm{N}(\%)$ & N (\%) & N (\%) & \\
\hline Heartburn & 449 & 595 & 752 & 749 & 411 & 362 & 1496 & 541 & 555 & 2959 \\
\hline \multirow{2}{*}{ sensation } & $(15.2)$ & $(20.1)$ & $(25.4)$ & $(25.3)$ & (13.9) & $(12.2)$ & $(50.6)$ & $(18.3)$ & (18.8) & $(31.9)$ \\
\hline & 874 & 882 & 1163 & 1209 & 587 & 425 & 2303 & 910 & 1072 & 4723 \\
\hline Regurgitation & $(18.5)$ & (18.7) & $(24.6)$ & $(25.6)$ & $(12.4)$ & $(9.0)$ & $(48.8)$ & (19.3) & $(22.7)$ & $(51.0)$ \\
\hline
\end{tabular}

Numbers may not add together to the total numbers due to a few missing data.

\section{Statistical analysis}

Data were cleaned and described by mean, standard deviation, frequency, and percentage. Univariate analyses were done applying Chi-square or Mann-Whitney U tests.

Variable selection was done according to univariate $P$ value of 0.1 . A multivariable logistic regression model was fitted to get adjusted odds ratios and 95\% confidence intervals for variables in relation to the GERD. A backward elimination approach was applied. $P$ value less than 0.05 was considered as statistically significant. Data analyses were done using SPSS software, version 21 (SPSS Inc., Chicago, IL, USA).

\section{RESULTS}

Of the participants in PCS, 4276 (46.2\%) were male and $4988(53.8 \%)$ were female. Mean age of the participants was $52.6 \pm 9.7$ years. Almost $32 \%(\mathrm{n}=2959)$ reported heartburn sensation and approximately $39.2 \%$ $(\mathrm{n}=1160)$ sensed heartburn weekly or daily (table 1$)$. But regurgitation was the most frequent symptom as about half of them $(n=4723)$ had regurgitation and 38\% $(\mathrm{n}=1796)$ reported this symptom as weekly or daily. Almost 2262 (24.4\%) participants had reported both symptoms (table 1).

Overall, 5420 (58.50\%, 95\% CI: 57.49 - 59.51) participants had heartburn sensation and/or regurgitation, but 2325 (25.10\%, 95\% CI: 24.22 - 25.99) participants had frequent GERD (weekly or more frequently). Also 218 (2.3\%) participants reported current use of some medications (proton pump inhibitors, $\mathrm{H}_{2}$ receptor blockers, and antacids). However, as they had neither heartburn sensation nor regurgitation, they were not considered as GERD positive cases.

The mean age of the participants with and without
GERD were $52.95 \pm 9.77$ and $52.21 \pm 9.56$ years, respectively $(P<0.001)$. GERD was significantly more prevalent in women compared with men $(58.90 \%$ vs. $41.10 \%, p<0.001)$.

According to univariate analysis there was a positive association between GERD and frequent GERD with being female; having higher age, higher BMI, higher waist to hip ratio, higher waist circumference, having low educational level, being divorced/widowed/separated, cigarette smoking, and using non-cigarette tobacco.

We found that being female, older ages, and having low educational level had a positive association with severe symptom sensation.

Having both symptoms (heartburn and regurgitation) was significantly associated with severity and frequency of symptoms.

Older ages, lower educational level, non-cigarette tobacco smoking, and opium consumption were significantly associated with frequent GERD in men. Also, older ages, higher BMI, abdominal obesity, being divorced/ widowed/separated, lower educational level, and noncigarette tobacco smoking were significantly associated with the presence of frequent GERD in women (table 2).

Positive history of underlying diseases including hypertension, anxiety, depression, and insomnia were significantly correlated with GERD, and frequent GERD. Diabetes mellitus had an association with GERD (table 3).

Sex, age, marital status, educational level, waist to hip ratio, hypertension, anxiety, depression, insomnia, cigarette smoking, and non-cigarette tobacco use were entered into the multivariable model. Older ages (OR $1.20,95 \%$ CI 1.058 - 1.36), female sex (OR 1.45, 95\% CI 1.27 - 1.65), divorce/widow/separation (OR 1.38, 95\% 


\section{2 Epidemiology of GERD in Southern Iran}

Table 2: Sex-stratified comparison of demographic, anthropometric, and lifestyle characteristics between the studied population with GERD and frequent GERD

\begin{tabular}{|c|c|c|c|c|c|c|c|c|c|c|}
\hline \multirow{3}{*}{ Characteristics } & \multicolumn{5}{|c|}{$\operatorname{Men}(n=4276)$} & \multicolumn{5}{|c|}{ Women $(n=4988)$} \\
\hline & All & $\begin{array}{c}\text { GERD } \\
(\mathrm{N}=2230)\end{array}$ & $P$ & $\begin{array}{c}\text { Frequent } \\
\text { GERD } \\
(\mathrm{N}=843)\end{array}$ & $P$ & All & $\begin{array}{c}\text { GERD } \\
(\mathrm{N}=3190)\end{array}$ & $P$ & $\begin{array}{c}\text { Frequent } \\
\text { GERD } \\
(\mathrm{N}=1482)\end{array}$ & $P$ \\
\hline & N (\%) & $\mathrm{N}(\%)$ & & N (\%) & & N (\%) & $\mathrm{N}(\%)$ & & $\mathrm{N}(\%)$ & \\
\hline Age* $^{*}$ & $\begin{array}{c}52.73 \pm \\
9.91\end{array}$ & $\begin{array}{l}52.69 \pm \\
10.03\end{array}$ & $0.534 \ddagger$ & $\begin{array}{c}53.54 \pm \\
10.27\end{array}$ & $0.015 \ddagger$ & $\begin{array}{c}52.57 \pm \\
9.49\end{array}$ & $\begin{array}{c}53.13 \pm \\
9.57\end{array}$ & $<0.001 \ddagger$ & $\begin{array}{l}53.75 \pm \\
9.95\end{array}$ & $<0.001 \ddagger$ \\
\hline BMI* & & & $0.434^{\bullet}$ & & $0.521^{\bullet}$ & & & $.012^{\bullet}$ & & $0.016^{\bullet}$ \\
\hline $\begin{array}{l}\text { Lower and } \\
\text { Normal weight }\end{array}$ & $\begin{array}{l}2442 \\
(57.4)\end{array}$ & $\begin{array}{c}1252 \\
(56.4)\end{array}$ & & $\begin{array}{l}497 \\
(59)\end{array}$ & & $\begin{array}{l}1661 \\
(33.5)\end{array}$ & $\begin{array}{l}1017 \\
(32)\end{array}$ & & $\begin{array}{c}458 \\
(31.1)\end{array}$ & \\
\hline Over weight & $\begin{array}{c}1428 \\
(33.6)\end{array}$ & $\begin{array}{c}759 \\
(34.2) \\
\end{array}$ & & $\begin{array}{c}269 \\
(31.9) \\
\end{array}$ & & $\begin{array}{l}2012 \\
(40.5)\end{array}$ & $\begin{array}{l}1326 \\
(41.8)\end{array}$ & & $\begin{array}{c}640 \\
(43.5)\end{array}$ & \\
\hline Obese & $386(9)$ & $\begin{array}{l}207 \\
(9.3)\end{array}$ & & $\begin{array}{l}76 \\
(9)\end{array}$ & & $\begin{array}{l}1289 \\
(26)\end{array}$ & $\begin{array}{c}831 \\
(26.2)\end{array}$ & & $\begin{array}{c}373 \\
(25.4)\end{array}$ & \\
\hline $\begin{array}{l}\text { Waist } \\
\text { circumference* }\end{array}$ & & & $0.962 \dagger$ & & $0.404 \dagger$ & & & $0.001 \uparrow$ & & $0.001 \dagger$ \\
\hline Normal & $\begin{array}{c}3772 \\
(88.2)\end{array}$ & $\begin{array}{c}1966 \\
(88.2)\end{array}$ & & $\begin{array}{c}751 \\
(89.1)\end{array}$ & & $\begin{array}{l}1714 \\
(34.4)\end{array}$ & $\begin{array}{c}1045 \\
(32.8)\end{array}$ & & $\begin{array}{l}459 \\
(31)\end{array}$ & \\
\hline At risk & $\begin{array}{c}504 \\
(11.8)\end{array}$ & $\begin{array}{c}264 \\
(11.8)\end{array}$ & & $\begin{array}{c}92 \\
(10.9)\end{array}$ & & $\begin{array}{c}3274 \\
(65.6)\end{array}$ & $\begin{array}{l}2145 \\
(67.2)\end{array}$ & & $\begin{array}{l}1023 \\
(69)\end{array}$ & \\
\hline $\begin{array}{l}\text { Waist to hip } \\
\text { ratio** }\end{array}$ & & & $0.025 \dagger$ & & $0.802 \uparrow$ & & & $0.016 \dagger$ & & $<0.001 \uparrow$ \\
\hline Normal & $\begin{array}{l}1294 \\
(30.4)\end{array}$ & $\begin{array}{c}641 \\
(28.9)\end{array}$ & & $\begin{array}{c}259 \\
(30.8)\end{array}$ & & $\begin{array}{l}548 \\
(11)\end{array}$ & $\begin{array}{c}325 \\
(10.2)\end{array}$ & & $\begin{array}{l}126 \\
(8.6)\end{array}$ & \\
\hline At risk & $\begin{array}{l}2964 \\
(69.6)\end{array}$ & $\begin{array}{l}1577 \\
(71.1)\end{array}$ & & $\begin{array}{c}583 \\
(69.2)\end{array}$ & & $\begin{array}{l}4414 \\
(89)\end{array}$ & $\begin{array}{l}2849 \\
(89.8)\end{array}$ & & $\begin{array}{l}1345 \\
(91.4)\end{array}$ & \\
\hline Ethnicity & & & $0.580 \dagger$ & & $0.069 \dagger$ & & & $0.061 \dagger$ & & $0.491 \dagger$ \\
\hline Fars & $\begin{array}{l}2368 \\
(55.4)\end{array}$ & $\begin{array}{c}1244 \\
(55.8)\end{array}$ & & $\begin{array}{c}443 \\
(52.6)\end{array}$ & & $\begin{array}{l}2849 \\
(57.1)\end{array}$ & $\begin{array}{c}1854 \\
(58.1)\end{array}$ & & $\begin{array}{c}835 \\
(56.3)\end{array}$ & \\
\hline Turk/others & $\begin{array}{c}1908 \\
(44.6)\end{array}$ & $\begin{array}{c}986 \\
(44.2)\end{array}$ & & $\begin{array}{c}400 \\
(47.4)\end{array}$ & & $\begin{array}{l}2139 \\
(42.9)\end{array}$ & $\begin{array}{l}1336 \\
(41.9)\end{array}$ & & $\begin{array}{c}647 \\
(43.7)\end{array}$ & \\
\hline Marital status & & & $0.302^{\bullet}$ & & $0.185^{\bullet}$ & & & $0.001^{\bullet}$ & & $<0.001^{\bullet}$ \\
\hline Single & $\begin{array}{l}50 \\
(1.2)\end{array}$ & $\begin{array}{c}27 \\
(1.2)\end{array}$ & & $\begin{array}{c}15 \\
(1.8)\end{array}$ & & $\begin{array}{l}247 \\
(5)\end{array}$ & $\begin{array}{c}142 \\
(4.5)\end{array}$ & & $\begin{array}{c}53 \\
(3.6)\end{array}$ & \\
\hline Married & $\begin{array}{l}4174 \\
(97.6)\end{array}$ & $\begin{array}{l}2171 \\
(97.4)\end{array}$ & & $\begin{array}{l}818 \\
(97)\end{array}$ & & $\begin{array}{l}4038 \\
(81)\end{array}$ & $\begin{array}{l}2559 \\
(80.2)\end{array}$ & & $\begin{array}{l}1165 \\
(78.6)\end{array}$ & \\
\hline $\begin{array}{l}\text { divorced/ } \\
\text { widowed/ } \\
\text { separated }\end{array}$ & $\begin{array}{c}51 \\
(1.2)\end{array}$ & $\begin{array}{l}32 \\
(1.4)\end{array}$ & & $\begin{array}{l}10 \\
(1.2)\end{array}$ & & $\begin{array}{c}701 \\
(14.1)\end{array}$ & $\begin{array}{c}488 \\
(15.3)\end{array}$ & & $\begin{array}{c}264 \\
(17.8)\end{array}$ & \\
\hline $\begin{array}{l}\text { Educational } \\
\text { level }\end{array}$ & & & $0.001^{\bullet}$ & & $0.001^{\bullet}$ & & & $0.014^{\bullet}$ & & $0.008^{\bullet}$ \\
\hline Illiterate & $\begin{array}{l}1336 \\
(31.3)\end{array}$ & $\begin{array}{c}678 \\
(30.5)\end{array}$ & & $\begin{array}{c}307 \\
(36.5)\end{array}$ & & $\begin{array}{c}3202 \\
(64.2)\end{array}$ & $\begin{array}{l}2083 \\
(65.3)\end{array}$ & & $\begin{array}{c}997 \\
(67.3)\end{array}$ & \\
\hline $\begin{array}{l}\leq \text { High school } \\
\text { diploma }\end{array}$ & $\begin{array}{l}2673 \\
(62.6)\end{array}$ & $\begin{array}{l}601 \\
(27)\end{array}$ & & $\begin{array}{c}494 \\
(58.7)\end{array}$ & & $\begin{array}{c}1765 \\
(35.4)\end{array}$ & $\begin{array}{c}971 \\
(30.5)\end{array}$ & & $\begin{array}{c}481 \\
(32.5)\end{array}$ & \\
\hline University & $\begin{array}{l}263 \\
(6.2)\end{array}$ & $\begin{array}{c}463 \\
(20.8) \\
\end{array}$ & & $\begin{array}{c}41 \\
(4.9)\end{array}$ & & $\begin{array}{c}18 \\
(0.4)\end{array}$ & $\begin{array}{l}96 \\
(3)\end{array}$ & & $\begin{array}{c}3 \\
(.2)\end{array}$ & \\
\hline $\begin{array}{l}\text { Cigarette } \\
\text { Smoking } \\
\text { (ever) }\end{array}$ & & & $0.165 \dagger$ & & $0.081 \dagger$ & & & $0.119 \dagger$ & & $0.999 \dagger$ \\
\hline Yes & $\begin{array}{c}1873 \\
(43.8)\end{array}$ & $\begin{array}{c}1000 \\
(44.8)\end{array}$ & & $\begin{array}{c}392 \\
(46.5)\end{array}$ & & $\begin{array}{c}45 \\
(0.9)\end{array}$ & $\begin{array}{c}34 \\
(1.1)\end{array}$ & & $\begin{array}{c}13 \\
(.9)\end{array}$ & \\
\hline No & $\begin{array}{c}2403 \\
(56.2)\end{array}$ & $\begin{array}{c}1230 \\
(55.2)\end{array}$ & & $\begin{array}{c}451 \\
(53.5)\end{array}$ & & $\begin{array}{c}4943 \\
(99.1)\end{array}$ & $\begin{array}{c}3156 \\
(98.9)\end{array}$ & & $\begin{array}{c}1469 \\
(99.1)\end{array}$ & \\
\hline $\begin{array}{l}\text { Use of } \\
\text { Non-cigarette } \\
\text { tobacco(ever) }\end{array}$ & & & $0.349 \dagger$ & & $0.004 \uparrow$ & & & $0.023 \dagger$ & & $0.002 \uparrow$ \\
\hline Yes & $\begin{array}{c}1231 \\
(28.8)\end{array}$ & $\begin{array}{c}649 \\
(29.1)\end{array}$ & & $\begin{array}{c}277 \\
(32.9)\end{array}$ & & $\begin{array}{c}2306 \\
(46.3)\end{array}$ & $\begin{array}{c}1514 \\
(47.5)\end{array}$ & & $\begin{array}{c}735 \\
(49.7)\end{array}$ & \\
\hline No & $\begin{array}{c}3038 \\
(71.2)\end{array}$ & $\begin{array}{c}1580 \\
(70.9)\end{array}$ & & $\begin{array}{c}566 \\
(67.1)\end{array}$ & & $\begin{array}{c}2672 \\
(53.7)\end{array}$ & $\begin{array}{c}1671 \\
(52.5)\end{array}$ & & $\begin{array}{c}743 \\
(50.3)\end{array}$ & \\
\hline
\end{tabular}

Middle East Journal of Digestive Diseases/ Vol.9/ No.3/July 2017 


\begin{tabular}{|c|c|c|c|c|c|c|c|c|c|c|}
\hline \multirow{3}{*}{ Characteristics } & \multicolumn{5}{|c|}{$\operatorname{Men}(n=4276)$} & \multicolumn{5}{|c|}{ Women $(n=4988)$} \\
\hline & All & $\begin{array}{l}\text { GERD } \\
(\mathrm{N}=2230)\end{array}$ & $P$ & $\begin{array}{c}\text { Frequent } \\
\text { GERD } \\
(\mathrm{N}=843)\end{array}$ & $P$ & All & $\begin{array}{c}\text { GERD } \\
(\mathrm{N}=3190)\end{array}$ & $P$ & $\begin{array}{c}\text { Frequent } \\
\text { GERD } \\
(\mathrm{N}=1482)\end{array}$ & $P$ \\
\hline & N (\%) & N (\%) & & N (\%) & & $\mathrm{N}(\%)$ & N (\%) & & $\mathrm{N}(\%)$ & \\
\hline $\begin{array}{l}\text { Opium abuse } \\
\text { (ever) }\end{array}$ & & & $0.069 \dagger$ & & $<0.001 \dagger$ & & & $0.999 \dagger$ & & $0.336 \dagger$ \\
\hline Yes & $\begin{array}{c}742 \\
(17.4) \\
\end{array}$ & $\begin{array}{c}410 \\
(18.4) \\
\end{array}$ & & $\begin{array}{c}195 \\
(23.1) \\
\end{array}$ & & $\begin{array}{c}32 \\
(0.6) \\
\end{array}$ & $\begin{array}{l}21 \\
(.7) \\
\end{array}$ & & $\begin{array}{c}12 \\
(.8) \\
\end{array}$ & \\
\hline No & $\begin{array}{l}3534 \\
(82.6)\end{array}$ & $\begin{array}{l}1820 \\
(81.6)\end{array}$ & & $\begin{array}{c}648 \\
(76.9)\end{array}$ & & $\begin{array}{l}4956 \\
(99.4)\end{array}$ & $\begin{array}{l}3169 \\
(99.3)\end{array}$ & & $\begin{array}{l}1470 \\
(99.2)\end{array}$ & \\
\hline $\begin{array}{l}\text { Use of alcohol } \\
\text { (ever) }\end{array}$ & & & $0.205 \dagger$ & & $0.319 \dagger$ & & & $0.570 \dagger$ & & $0.690 \dagger$ \\
\hline Yes & $\begin{array}{l}166 \\
(3.9)\end{array}$ & $\begin{array}{l}95 \\
(4.3)\end{array}$ & & $\begin{array}{c}38 \\
(4.5)\end{array}$ & & $\begin{array}{c}30 \\
(0.6)\end{array}$ & $\begin{array}{l}21 \\
(0.7)\end{array}$ & & $\begin{array}{l}10 \\
(.7)\end{array}$ & \\
\hline No & $\begin{array}{l}4110 \\
(96.1)\end{array}$ & $\begin{array}{l}2135 \\
(95.7)\end{array}$ & & $\begin{array}{c}805 \\
(95.5)\end{array}$ & & $\begin{array}{l}4958 \\
(99.4)\end{array}$ & $\begin{array}{l}3169 \\
(99.3)\end{array}$ & & $\begin{array}{l}1472 \\
(99.3)\end{array}$ & \\
\hline
\end{tabular}

Numbers may not add together to the total numbers due to missing data. GERD: gastroesophageal reflux disease; BMI: body mass index. * For age, the values are (mean \pm standard deviation) years and $P$ was calculated by Mann-Whitney $\mathrm{U}$ test. * Frequent GERD means having at least weekly symptoms. BMI $<18.5$ is underweight, from 18.5 to 24.9 is normal weight, from 25 to 29.9 is overweight, and $\geq 30$ is obesity. Waist circumference $>102$ centimeters for men and $>88$ centimeters for women and also waist to hip ratio $>0.90$ for men and $>0.85$ for women are at risk. $P$ was calculated by: $\ddagger$ Mann-Whitney $\mathrm{U}$ test, ${ }^{\circ} \mathrm{X}^{2}$ test and $\dagger$ fisher exact test.

Table 3: Comparison of the history of underlying diseases between the participants with and without GERD and frequent GERD

\begin{tabular}{|c|c|c|c|c|c|c|}
\hline History of underlying & $\begin{array}{l}\text { All GERD } \\
(\mathbf{N}=\mathbf{5 4 2 0})\end{array}$ & $\begin{array}{l}\text { No GERD } \\
(\mathrm{N}=3844)\end{array}$ & $P \dot{\dagger}$ & $\begin{array}{l}\text { Frequent* GERD } \\
(\mathbf{N}=\mathbf{2 3 2 5})\end{array}$ & $\begin{array}{c}\text { No Frequent } \\
\text { GERD }(\mathbf{N}=6939)\end{array}$ & $P_{\dagger}^{\dagger}$ \\
\hline & $\mathrm{N}(\%)$ & N (\%) & & N (\%) & N (\%) & \\
\hline Hypertension & & & $<0.001$ & & & $<0.001$ \\
\hline Yes & $996(18.4)$ & $517(13.4)$ & & $478(20.6)$ & $1035(14.9)$ & \\
\hline No & 4424 (81.6) & $3327(86.6)$ & & $1847(79.4)$ & $5904(85.1)$ & \\
\hline Diabetes mellitus & & & 0.007 & & & 0.151 \\
\hline Yes & $549(10.1)$ & $325(8.5)$ & & $237(10.2)$ & $637(9.2)$ & \\
\hline No & $4871(89.9)$ & 3519 (91.5) & & $2088(89.8)$ & $6302(90.8)$ & \\
\hline Anxiety & & & $<0.001$ & & & $<0.001$ \\
\hline Yes & $1980(36.5)$ & 764 (19.9) & & $850(36.6)$ & $1894(27.3)$ & \\
\hline No & $3440(63.5)$ & $3074(80.1)$ & & $1475(63.4)$ & $5045(72.7)$ & \\
\hline Depression & & & $<0.001$ & & & $<0.001$ \\
\hline Yes & $1291(23.8)$ & $504(13.1)$ & & $566(24.3)$ & $1229(17.7)$ & \\
\hline No & $4129(76.2)$ & $3340(86.9)$ & & $1759(75.7)$ & $5710(82.3)$ & \\
\hline Insomnia & & & $<0.001$ & & & $<0.001$ \\
\hline Yes & $1260(23.2)$ & 535 (13.9) & & $596(25.6)$ & $1199(17.3)$ & \\
\hline No & $4160(76.8)$ & $3309(86.1)$ & & $1729(74.4)$ & $5740(82.7)$ & \\
\hline
\end{tabular}

Numbers may not add together to the total numbers due to missing data. GERD: gastroesophageal reflux disease. * Frequent GERD means having at least weekly symptoms. $\dagger P$ was calculated by: fisher exact test.

CI 1.01 - 1.91), and non-cigarette tobacco smoking (OR $1.14,95 \%$ CI 1.03 - 1.26) had correlation with frequent GERD. Also, the participants with history of hypertension (OR 1.16, 95\% CI 1.02 - 1.33), anxiety (OR 1.24, 95\% CI 1.11 - 1.40), and insomnia (OR 1.36, 95\% CI 1.21 - 1.54) were more likely to have frequent GERD (table 4).

\section{DISCUSSION}

In this study, $25.1 \%$ of 40 - 75 years old participants had GERD symptoms weekly or more frequently. We also showed that $58.5 \%$ of all the participants had experienced GERD symptoms at least once in the last 12 months. There were strong associations between frequent GERD and fe- 
Table 4: Association of covariates with frequent GERD

\begin{tabular}{|c|c|c|c|c|}
\hline \multirow{2}{*}{ Variables } & \multirow{2}{*}{$\begin{array}{c}\begin{array}{c}\text { Frequent* } \\
\text { GERD (N=2325) }\end{array} \\
\mathrm{N}(\%)\end{array}$} & \multirow{2}{*}{$\begin{array}{c}\text { No Frequent } \\
\text { GERD }(\mathbf{N}=6939)\end{array}$} & \multicolumn{2}{|c|}{ OR $(95 \%$ CI) } \\
\hline & & & Unadjusted & Adjusted \\
\hline \multicolumn{5}{|l|}{ Age* } \\
\hline $40-60$ years & $610(26.2)$ & $1390(20)$ & Reference & Reference \\
\hline $61^{+}$ & $1715(73.8)$ & $5549(80)$ & $1.42(1.27-1.58)$ & $1.20(1.06-1.36)$ \\
\hline \multicolumn{5}{|l|}{ Sex } \\
\hline Male & $843(36.3)$ & $3433(49.5)$ & Reference & Reference \\
\hline Female & $1482(63.7)$ & $3506(50.5)$ & $1.72(1.56-1.89)$ & $1.45(1.27-1.65)$ \\
\hline \multicolumn{5}{|l|}{ Marital status } \\
\hline Single & $68(2.9)$ & $229(3.3)$ & Reference & Reference \\
\hline Married & $1983(85.3)$ & $6229(89.9)$ & $1.07(.81-1.41)$ & $1.12(.84-1.48)$ \\
\hline Divorced/ widowed/ separated & $274(11.8)$ & $478(6.9)$ & $1.93(1.42-2.63)$ & $1.38(1.01-1.91)$ \\
\hline \multicolumn{5}{|l|}{ Educational level } \\
\hline Illiterate & $1304(56.1)$ & 3234 (46.6) & $2.17(1.56-3.01)$ & $1.43(1.02-2.03)$ \\
\hline$\leq$ High school diploma & $975(42)$ & $3463(49.9)$ & $1.51(1.09-2.11)$ & $1.29(.92-1.81)$ \\
\hline University & $44(1.9)$ & $237(3.4)$ & Reference & Reference \\
\hline \multicolumn{5}{|l|}{ Waist to hip ratio* } \\
\hline Normal & $385(16.6)$ & $1457(21.1)$ & Reference & Reference \\
\hline At risk & $1928(83.4)$ & $5448(78.9)$ & $1.34(1.18-1.52)$ & $1.12(.98-1.28)$ \\
\hline \multicolumn{5}{|l|}{ Hypertension } \\
\hline Yes & $478(20.6)$ & $1035(14.9)$ & $1.48(1.31-1.66)$ & $1.16(1.02-1.33)$ \\
\hline No & $1847(79.4)$ & $5904(85.1)$ & Reference & Reference \\
\hline \multicolumn{5}{|l|}{ Anxiety } \\
\hline Yes & $850(36.6)$ & $1894(27.3)$ & $1.53(1.39-1.69)$ & $1.24(1.11-1.40)$ \\
\hline No & $1475(63.4)$ & $5045(72.7)$ & Reference & Reference \\
\hline \multicolumn{5}{|l|}{ Depression } \\
\hline Yes & $566(24.3)$ & $1229(17.7)$ & $1.49(1.33-1.67)$ & $1.08(.95-1.24)$ \\
\hline No & $1759(75.7)$ & $5710(82.3)$ & Reference & Reference \\
\hline \multicolumn{5}{|l|}{ Insomnia } \\
\hline Yes & $596(25.6)$ & $1199(17.3)$ & $1.65(1.47-1.85)$ & $1.36(1.21-1.54)$ \\
\hline No & $1729(74.4)$ & $5740(82.7)$ & Reference & Reference \\
\hline \multicolumn{5}{|l|}{ Cigarette Smoking (ever) } \\
\hline Yes & $405(17.4)$ & $1513(21.8)$ & $.76(.67-85)$ & $1.13(.97-1.30)$ \\
\hline No & $1920(82.6)$ & $5426(78.2)$ & Reference & Reference \\
\hline \multicolumn{5}{|c|}{ Usage of Non-cigarette tobacco (ever) } \\
\hline Yes & $1012(43.6)$ & $2525(36.5)$ & $1.35(1.22-1.48)$ & $1.14(1.03-1.26)$ \\
\hline No & $1309(56.4)$ & $4401(63.5)$ & Reference & Reference \\
\hline
\end{tabular}

* Frequent GERD means having at least weekly symptoms. GERD: gastroesophageal reflux disease. * For age, the values are (mean \pm standard deviation) years and $P$ was calculated by Mann-Whitney U test. * Waist to hip ratio $>0.90$ for men and $>0.85$ for women are at risk.

male sex, and positive history of anxiety and insomnia.

The prevalence of frequent GERD in our study was close to that in western countries and Iran, but it was higher than East Asia. ${ }^{9,28,32}$ It may be due to more west- ernized lifestyle in the target population. ${ }^{33,34}$

In our study, about $32 \%$ of the participants reported heartburn sensation and $51 \%$ of them had regurgitation. Almost $24.4 \%$ of the participants had reported both 
symptoms. Consistent with previous studies, regurgitation was more common than heartburn in this study. ${ }^{1,2,10,27,35,36}$ Our data also showed that about half of the participants had moderate GERD symptoms similar to some of previous studies. ${ }^{10,16}$

In our study, older ages had an association with frequent GERD and sensing severe symptoms. This finding is consistent with some previous reports. ${ }^{9,12,13}$ This relationship may be due to the decrease in defense mechanisms against reflux i.e. the decrease in pressure of the lower esophageal sphincter. However, there are controversies regarding the association between increased age and frequent GERD as some studies have shown an increase in the incidence of GERD by increasing age up to 60 - 69 years and then a decrease. ${ }^{12,13,16,18}$

Unlike most of previous studies, our findings showed that male sex was a protective factor for the presence and severity of GERD symptoms. ${ }^{3,9,16,18,28,37}$ Higher prevalence among women may be due to higher prevalence of obesity in women compared with men. ${ }^{37-39}$ It also may be due to the effects of female sex hormones on the lower esophageal sphincter. ${ }^{37,39,40}$

Among the participants with abdominal obesity, GERD and frequent GERD were more common. Many studies reported a significant association between GERD and abdominal obesity. It is probably because of hiatal hernia and increase in intra-abdominal pressure among patients with central obesity. In addition to central obesity, previous studies have shown that BMI and weight cycling could be associated with GERD. ${ }^{3,9,28,37,40-42}$

We found that frequency and severity of GERD were associated with marital status because frequent GERD was more common among divorced/widowed/separated participants compared with singles and married ones (OR 1.38, 95\% CI 1.01 - 1.91). It maybe resulted from a higher prevalence of depression and anxiety among divorced/widowed/separated participants. It may also be also due to considerable changes in dietary pattern. ${ }^{32,43-45}$

Consistent with the findings of some previous studies like those conducted in Albania, India, and Iran, the frequency and severity of GERD symptoms in participants with lower educational level was significantly higher than those with higher educational level. , $16,28,46^{2}$

In both men and women, non-cigarette tobacco smoking had an association with GERD (OR 1.14, 95\%
CI 1.03 - 1.26). There is also some evidence (reduce the LES pressure and salivary secretion of bicarbonates) showing that non-cigarette tobacco use is correlated with GERD presence, but some other studies did not show such a relationship. ${ }^{8,28,38,45-47}$

In contrast to some studies, we found that there was an association between opium consumption and GERD. ${ }^{8,16,28,46}$ Despite this, after sex stratification, opium had only an association with frequent GERD in men.

Sharma and colleagues showed a positive relationship between hypertension and GERD. ${ }^{48}$ In our study, we found that having a positive history of hypertension was related to GERD, frequent GERD, and having severe GERD symptoms.

In this study, having a history of diabetes mellitus (DM) had a significant association with GERD in univariate analysis, although this association was not confirmed in multivariable analysis. One study in the United States has shown that approximately $41 \%$ of the patients with diabetes had GERD symptoms. ${ }^{49,50}$ Also, a meta-analysis has indicated the higher prevalence of GERD in patients with DM than general population. Association of obesity and metabolic syndrome could be considered as a potential justification for the relationship between GERD symptoms and type II DM. Peripheral neuropathy as a consequence of type II DM may be another reason for this relationship. ${ }^{42,50-56}$

Previous studies showed bidirectional association between GERD and psychological problems such as anxiety, depression, sleep disturbance, and insomnia..$^{32,57-59}$ In our results, positive history of anxiety (OR 1.24, 95\% CI 1.11 - 1.40) and insomnia (OR 1.36, 95\% CI 1.21 - 1.54) were significantly associated with GERD and frequent GERD.

\section{Strengths and Limitations of the Study}

The strengths of this study are large sample size, and availability of many lifestyle factors, some of which have not been previously studied.

We also cross-validated the specificity of our prevalence estimate, considering the current use of medications, which could result in GERD symptom relief for cases who did not have heartburn sensation or regurgitation symptoms. As less than $2.3 \%$ of the participants reported the use of interested medications, our estimated prevalence may be a little increased if these cases considered as GERD positive. 
The main limitation of this study is the cross-sectional nature of the study, which may not be able to determine the causal relationship between exposures and outcomes. Therefore, we should be cautious in the interpretation of the results. Also, we studied the data of a cohort on 40 - 75 years old people, so we cannot generalize the results to all population.

In conclusion, one out of four middle aged people in rural population suffer from GERD. We found that some factors such as female sex, higher age, higher BMI, having abdominal obesity, being divorced/widowed/separated, having low educational level, using non-cigarette tobacco, and history of hypertension, anxiety, and insomnia are associated with prevalent GERD.

\section{ACKNOWLEDGEMENTS}

The present article was extracted from the MPH thesis written by Zohre Khodamoradi and was financially supported by Vice Chancellery for Research in Shiraz University of Medical Sciences. The authors would like to thank Dr. Nasrin Shokrpour for editorial assistance.

\section{CONFLICT OF INTEREST}

The authors declare no conflict of interest related to this work.

\section{REFERENCES}

1. Pourshams A, Rahmani A, Hatami K. Gastroesophageal reflux disease in Iran. Govaresh 2005;10:48-53.

2. Somi MH, Farhang S, Mirinezhad K, Jazayeri E, Nasseri-Moghaddam S, Moayeri S, et al. Prevalence and precipitating factors of gastroesophageal reflux disease in a young population of Tabriz, Northwest of Iran. Saudi Med J 2006;27:1878-81.

3. Feldman M, Friedman LS, Brandt LJ. Sleisenger and Fordtran's Gastrointestinal and Liver Disease: Pathophysiology, Diagnosis, Management: Saunders/Elsevier; 2015.

4. Badillo R, Francis D. Diagnosis and treatment of gastroesophageal reflux disease. World J Gastrointest Pharmacol Ther 2014;5:105-12. doi: 10.4292/wjgpt.v5.i3.105.

5. Tan VP, Wong BC, Wong WM, Leung WK, Tong D, Yuen MF, et al. Gastroesophageal Reflux Disease: Cross-Sectional Study Demonstrating Rising Prevalence in a Chinese Population. J Clin Gastroenterol 2016;50:e1-7. doi: 10.1097/MCG.0000000000000304.

6. Islami F, Pourshams A, Nasseri-Moghaddam S, Khademi H, Poutschi H, Khoshnia M, et al. Gastroesophageal Reflux Disease and overall and Cause-specific Mortality: A
Prospective Study of 50000 Individuals. Middle East J Dig Dis 2014;6:65. doi: 10.1016/S0016-5085(14)63119-X.

7. Sepanlou S, Khademi H, Abdollahzadeh N, Noori F, Malekzadeh F, Malekzadeh R. Time Trends of Gastro-esophageal Reflux Disease (GERD) and Peptic Ulcer Disease (PUD) in Iran. Middle East J Dig Dis 2010;2:78-83.

8. Islami F, Nasseri-Moghaddam S, Pourshams A, Poustchi H, Semnani S, Kamangar F, et al. Determinants of gastroesophageal reflux disease, including hookah smoking and opium use- a cross-sectional analysis of 50,000 individuals. PLoS One 2014;9:e89256. doi: 10.1371/journal. pone.0089256.

9. Podolsky DK, Camilleri M, Fitz JG, Kalloo AN, Shanahan F, Wang TC. Yamada's Textbook of Gastroenterology, 2 Volume Set: Wiley 2015.

10. Nouraie M, Razjouyan H, Assady M, Malekzadeh R, Nasseri-Moghaddam S. Epidemiology of gastroesophageal reflux symptoms in Tehran, Iran: a population-based telephone survey. Arch Iran Med 2007;10:289-94. doi: 07103/AIM.003.

11. Delavari A, Moradi G, Birjandi F, Elahi E, Saberifiroozi M. The Prevalence of Gastroesophageal Reflux Disease (GERD) in the Islamic Republic of Iran: A Systematic Review. Middle East J Dig Dis 2012;4:5-15.

12. El-Serag HB, Sweet S, Winchester CC, Dent J. Update on the epidemiology of gastro-oesophageal reflux disease: a systematic review. Gut 2014;63:871-80. doi: 10.1136/ gutjnl-2012-304269.

13. Boeckxstaens G, El-Serag HB, Smout AJ, Kahrilas PJ. Symptomatic reflux disease:1185-93. doi:10.1136/ gutjnl-2013-306393.

14. Nouraie M, Radmard AR, Zaer-Rezaii H, Razjouyan H, Nasseri-Moghaddam S, Malekzadeh R. Hygiene could affect GERD prevalence independently: a population-based study in Tehran. Am J Gastroenterol 2007;102:1353-60. doi: 10.1111/j.1572-0241.2007.01208.x.

15. Masoumi SJ, Khademolhosseini F, Mehrabani D, Moradi F, Mostaghni AA, Zare N, et al. Correlation of quality of life with gastroesophageal reflux disease amongst Qashqai nomads in Iran. Arch Iran Med 2012;15:747-50. doi: 0121512/AIM.005.

16. Nasseri-Moghaddam S, Mofid A, Ghotbi MH, Razjouyan $\mathrm{H}$, Nouraie M, Ramard AR, et al. Epidemiological study of gastro-oesophageal reflux disease: reflux in spouse as a risk factor. Aliment Pharmacol Ther 2008;28:144-53. doi: 10.1111/j.1365-2036.2008.03708.x.

17. Wong BC, Kinoshita Y. Systematic review on epidemiology of gastroesophageal reflux disease in Asia. Clin Gastroenterol Hepatol 2006;4:398-407. doi: 10.1016/j. cgh.2005.10.011.

18. Bor S, Lazebnik L, Kitapcioglu G, Manannikof I, Vasiliev Y. Prevalence of gastroesophageal reflux disease in Moscow. Dis Esophagus 2016:159-65. doi: 10.1111/dote.12310.

19. Jung HK. Epidemiology of gastroesophageal reflux disease in Asia: a systematic review. J Neurogastroenterol 
Motil 2011;17:14-27. doi: 10.5056/jnm.2011.17.1.14.

20. Wang R, Yan X, Ma X-Q, Cao Y, Wallander MA, Johansson $\mathrm{S}$, et al. Burden of gastroesophageal reflux disease in Shanghai, China. Dig Liver Dis 2009;41:110-5. doi: 10.1016/j.dld.2008.06.014

21. Wong W, Lai K, Lam K, Hui W, Hu W, Lam C, et al. Prevalence, clinical spectrum and health care utilization of gastro-oesophageal reflux disease in a Chinese population: a population-based study. Aliment Pharmacol Ther 2003;18:595-604. doi: 10.1046/j.13652036.2003.01737.x

22. Cho YS, Choi MG, Jeong JJ, Chung WC, Lee IS, Kim $\mathrm{SW}$, et al. Prevalence and clinical spectrum of gastroesophageal reflux: a population-based study in Asansi, Korea. Am J Gastroenterol 2005;100:747-53. doi: 10.1111/j.1572-0241.2005.41245.x.

23. Kahrilas PJ. Diagnosis of symptomatic gastroesophageal reflux disease. Am J Gastroenterol 2003;98:S15-23. doi:10.1016/S0002-9270(03)00011-X.

24. Nasseri-Moghaddam S, Malekzadeh R, Sotoudeh M, Tavangar M, Azimi K, Sohrabpour AA, et al. Lower esophagus in dyspeptic Iranian patients: a prospective study. J Gastroenterol Hepatol 2003;18:315-21. doi: 10.1046/j.1440-1746.2003.02969.x.

25. Manterola C, Munoz S, Grande L, Bustos L. Initial validation of a questionnaire for detecting gastroesophageal reflux disease in epidemiological settings. $J$ Clin Epidemiol 2002;55:1041-5. doi:10.1016/S0895-4356(02)00454-7.

26. Katz PO, Gerson LB, Vela MF. Guidelines for the diagnosis and management of gastroesophageal reflux disease. Am J Gastroenterol 2013;108:308-28; quiz 29. doi: 10.1038/ajg.2012.444.

27. Bor S, Mandiracioglu A, Kitapcioglu G, Caymaz-Bor C, Gilbert RJ. Gastroesophageal reflux disease in a low-income region in Turkey. Am J Gastroenterol 2005; 100:75965. doi: 10.1111/j.1572-0241.2005.41065.x.

28. Wang HY, Leena KB, Plymoth A, Hergens MP, Yin L, Shenoy KT, et al. Prevalence of gastro-esophageal reflux disease and its risk factors in a community-based population in southern India. BMC Gastroenterol 2016;16:36. doi: 10.1186/s12876-016-0452-1.

29. Gandomkar A, Poustchi H, Moini M, Moghadami M, Imanieh H, Fattahi MR, et al. Pars cohort study of noncommunicable diseases in Iran: protocol and preliminary results. Int J Public Health 2017;62:397-406. doi: 10.1007/s00038-016-0848-2.

30. Obesity: preventing and managing the global epidemic: Report of a WHO consultation.World Health Organ Tech Rep Ser 2000;894:i-xii, 1-253.

31. Consultation WE. Waist circumference and waist-hip ratio. Report of a WHO Expert Consultation Geneva: World Health Organization. 2008:8-11.

32. Aletaha N, Pourshams A, Nouraie S, Malekzadeh R. The Role of Psychosocial Disorders in Gastroesophageal Reflux Disease. Govaresh 2007;12:92-7.
33. Ahmadi B, Alimohammadian M, Yaseri M, Majidi A, Boreiri M, Islami F, et al. Multimorbidity: Epidemiology and Risk Factors in the Golestan Cohort Study, Iran: A Cross-Sectional Analysis. Medicine (Baltimore). 2016;95:e2756. doi: 10.1097/MD.0000000000002756.

34. Rafiemanesh H, Maleki F, Mohammadian-Hafshejani A, Salemi M, Salehiniya H. The Trend in Histological Changes and the Incidence of Esophagus Cancer in Iran (2003-2008). Int J Prev Med 2016;7:31. doi: 10.4103/2008-7802.175990.

35. Delaney BC. Review article: prevalence and epidemiology of gastro-oesophageal reflux disease. Aliment Pharmacol Ther 2004;20 Suppl 8:2-4. doi: 10.1111/j.13652036.2004.02219.x.

36. Wang JH, Luo JY, Dong L, Gong J, Tong M. Epidemiology of gastroesophageal reflux disease: a general population-based study in Xi'an of Northwest China. World $J$ Gastroenterol 2004;10:1647-51. doi:10.3748/wjg.v10. i11.1647.

37. Chen Z, Thompson SK, Jamieson GG, Devitt PG, Watson DI. Effect of sex on symptoms associated with gastroesophageal reflux. Arch Surg 2011;146:1164-9. doi: 10.1001/archsurg.2011.248.

38. Nilsson M, Johnsen R, Ye W, Hveem K, Lagergren J. Lifestyle related risk factors in the aetiology of gastrooesophageal reflux. Gut 2004;53:1730-5. doi: 10.1136/ gut.2004.043265.

39. Hampel H, Abraham NS, El-Serag HB. Meta-analysis: obesity and the risk for gastroesophageal reflux disease and its complications. Ann Intern Med 2005;143:199211. doi: 10.7326/0003-4819-143-3-200508020-00006.

40. Nilsson M, Johnsen R, Ye W, Hveem K, Lagergren J. Obesity and estrogen as risk factors for gastroesophageal reflux symptoms. JAMA 2003;290:66-72. doi:10.1001/ jama.290.1.66

41. Jacobson BC, Somers SC, Fuchs CS, Kelly CP, Camargo Jr CA. Body-mass index and symptoms of gastroesophageal reflux in women. $N$ Engl $J$ Med 2006;354:2340-8 doi: 10.1056/NEJMoa054391.

42. Sun XM, Tan JC, Zhu Y, Lin L. Association between diabetes mellitus and gastroesophageal reflux disease: A meta-analysis. World J Gastroenterol 2015;21:3085-92. doi: 10.3748/wjg.v21.i10.3085.

43. Chen M, Xiong L, Chen $\mathrm{H}, \mathrm{Xu} \mathrm{A}, \mathrm{He} \mathrm{L}, \mathrm{Hu}$ P. Prevalence, risk factors and impact of gastroesophageal reflux disease symptoms: a population-based study in South China. Scand J Gastroenterol 2005;40:759-67. doi: $10.1080 / 00365520510015610$.

44. Lee S, Cho E, Grodstein F, Kawachi I, Hu FB, Colditz GA. Effects of marital transitions on changes in dietary and other health behaviours in US women. Int $J$ Epidemiol 2005;34:69-78. doi: 10.1093/ije/dyh258.

45. Festi D, Scaioli E, Baldi F, Vestito A, Pasqui F, Di Biase AR, et al. Body weight, lifestyle, dietary habits and gastroesophageal reflux disease. World $J$ Gastroentero. 
2009;15:1690-701. doi: 10.3748/wjg.15.1690.

46. Zheng Z, Nordenstedt H, Pedersen NL, Lagergren J, Ye W. Lifestyle factors and risk for symptomatic gastroesophageal reflux in monozygotic twins. Gastroenterology 2007;132:87-95. doi: 10.1053/j.gastro.2006.11.019.

47. Malekzadeh R, Nasseri-Moghaddam S, Sotoudeh M. Gastroesophageal reflux disease: the new epidemic. Arch Iranian Med 2003;6:127-40.

48. Sharma PK, Ahuja V, Madan K, Gupta S, Raizada A, Sharma MP. Prevalence, severity, and risk factors of symptomatic gastroesophageal reflux disease among employees of a large hospital in northern India. Indian Journal of Gastroenterology 2011;30:128-34. doi: 10.1007/ s12664-010-0065-5.

49. Wang X, Pitchumoni CS, Chandrarana K, Shah N. Increased prevalence of symptoms of gastroesophageal reflux diseases in type 2 diabetics with neuropathy. World $J$ Gastroenterol 2008;14:709-12. doi: 10.3748/wjg.14.709.

50. Ha JO, Lee TH, Lee CW, Park JY, Choi SH, Park HS, et al. Prevalence and Risk Factors of Gastroesophageal Reflux Disease in Patients with Type 2 Diabetes Mellitus. Diabetes Metab J 2016;40:297-307. doi: 10.4093/ dmj.2016.40.4.297.

51. Nishida T, Tsuji S, Tsujii M, Arimitsu S, Sato T, Haruna $\mathrm{Y}$, et al. Gastroesophageal reflux disease related to diabetes: Analysis of 241 cases with type 2 diabetes mellitus. J Gastroenterol Hepatol 2004;19:258-65. doi: 10.1111/j.1440-1746.2003.03288.x.

52. Lluch I, Ascaso JF, Mora F, Minguez M, Pena A, Hernan$\operatorname{dez} \mathrm{A}$, et al. Gastroesophageal reflux in diabetes mellitus. Am J Gastroenterol 1999;94:919-24. doi: 10.1111/j.15720241.1999.987 j.x.

53. Sun H, Yi L, Wu P, Li Y, Luo B, Xu S. Prevalence of Gastroesophageal Reflux Disease in Type II Diabetes Mellitus. Gastroenterol Res Pract 2014;2014:601571. doi: $10.1155 / 2014 / 601571$.

54. Fujiwara M, Miwa T, Kawai T, Odawara M. Gastroesophageal reflux disease in patients with diabetes: Preliminary study. $J$ Gastroenterol Hepatol 2015;30 Suppl 1:31-5. doi: 10.1111/jgh.12777.

55. Ke M. How is the autonomic nerve function different between gastroesophageal reflux disease alone and gastroesophageal reflux disease with diabetes mellitus neuropathy? J Neurogastroenterol Motil 2011;17:430-1;author reply 432-3. doi: 10.5056/jnm.2011.17.4.430.

56. Lee SD, Keum B, Chun HJ, Bak Y-T. Gastroesophageal reflux disease in type II diabetes mellitus with or without peripheral neuropathy. Journal of neurogastroenterology and motility 2011;17:274. doi: 10.5056\%2Fjnm.2011.17.3.274.

57. Kimura Y, Kamiya T, Senoo K, Tsuchida K, Hirano A, Kojima H, et al. Persistent reflux symptoms cause anxiety, depression, and mental health and sleep disorders in gastroesophageal reflux disease patients. $J$ Clin Biochem Nutr 2016;59:71-7. doi: 10.3164/jcbn.16-9.

58. Yang X-J, Jiang H-M, Hou X-H, Song J. Anxiety and de- pression in patients with gastroesophageal reflux disease and their effect on quality of life. World J Gastroenterol 2015;21:4302-09. doi: 10.3748\%2Fwjg.v21.i14.4302.

59. Kessing BF, Bredenoord AJ, Saleh CM, Smout AJ. Effects of anxiety and depression in patients with gastroesophageal reflux disease. Clinical gastroenterology and hepatology. the official clinical practice journal of the American Gastroenterological Association 2015;13:1089-95 e1. doi: 10.1016/j.cgh.2014.11.034. 